\title{
Clusters of Toxoplasmosis in Ganghwa-gun, Cheorwon-gun, and Goseong-gun, Korea
}

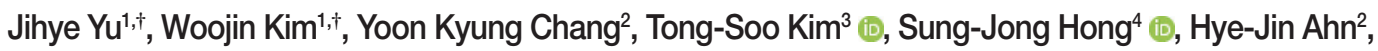 \\ Ho-Woo Nam², Dongjae Kim ${ }^{1, *}$ (i) \\ ${ }^{1}$ Department of Biomedicine Health Science, College of Medicine, The Catholic University of Korea, Seoul 06591, Korea; ${ }^{2}$ Department of \\ Parasitology, College of Medicine, The Catholic University of Korea, Seoul 06591, Korea; ${ }^{3}$ Department of Parasitology and Tropical Medicine and \\ Inha Research Institute for Medical Sciences, Inha University School of Medicine, Incheon 22333, Korea; ${ }^{4}$ Department of Medical Environmental \\ Biology, Chung-Ang University College of Medicine, Seoul 06974, Korea
}

\begin{abstract}
We find out the clusters with high toxoplasmosis risk to discuss the geographical pattern in Gyodong-myeon and Samsan-myeon of Ganghwa-gun, Cheorwon-gun, and Goseong-gun, Korea. Seroepidemiological data of toxoplasmosis surveyed using rapid diagnostic tests for the residents in the areas in 2019 were analyzed to detect clusters of the infection. The cluster was investigated using the SaTScan program which is based on Kulldorff's scan statistic. The clusters were found with $P$-values in each region analyzed in the program, and the risk and patient incidence of specific areas can be examined by the values such as relative risk and log likelihood ratio. Jiseok-ri and Insa-ri were found to be a cluster in Gyodong-myeon and Seokmo-ri was the cluster in Samsan-myeon. Yangji-ri and lgil-ri were found to be a cluster in Cheorwon-gun and Madal-ri and Baebong-ri were the cluster in Goseong-gun. This findings can be used to monitor and prevent toxoplasmosis infections occurring in vulnerable areas.
\end{abstract}

Key words: Toxoplasmosis, RDT, cluster, resident, Ganghwa-gun, Cheorwon-gun, Goseong-gun

\section{INTRODUCTION}

Toxoplasma gondii, an apicomplexan zoonotic protozoan, is one of the most widespread parasites worldwide. Warm blooded animals are its hosts, especially felids. Toxoplasmosis is acquired by the infection with $T$. gondii through undercooked meat, contaminated water, or mother to her fetus. Congenital toxoplasmosis may cause critical damages to the fetus [1-5]. For adult humans, most of their symptoms are light or asymptomatic. However, the infections can be exacerbated into chronic conditions and can cause serious diseases in immune compromised patients [6-9]. Rapid diagnostic test (RDT) is a tool for diagnosis of toxoplasmosis. Seroprevalence of toxoplasmosis in certain areas is surging recently [10-14]. Previous studies have shown that the overall proportion, gender and age distribution of Gyodong-myeon, Samsan-myeon and Cheorwon-gun [11-13]. In addition to this, if clusters with

- Received 30 March 2021, revised 17 May 2021, accepted 22 May 2021

*Corresponding author (djkim@catholic.ac.kr)

${ }^{\dagger}$ These authors contributed equally to this work.

(c) 2021, Korean Society for Parasitology and Tropical Medicine

This is an Open Access article distributed under the terms of the Creative Commons Attribution Non-Commercial License (https://creativecommons.org/licenses/by-nc/4.0 which permits unrestricted non-commercial use, distribution, and reproduction in any medium, provided the original work is properly cited. high risk are specified by comparing them to each region, efficient monitoring and health policies can be established accordingly.

Spatial scan statistic and Kulldorffs SaTScan Software were used to detect the clusters. SaTScan perform geographical analysis of disease to detect spatial or spatial-time clusters and to find if they are statistically significant. Generate scan statistics with observed and expected number of patients and find the cluster with 999 simulations within a predetermined window size via SaTScan. This study analyzed clusters using RDT for the detection of anti-T. gondii antibodies with the sera of residents in Gyodong-myeon, Samsam-myeon both in Ganghwagun, Incheon, Korea in 2019.

\section{MATERIALS AND METHODS}

\section{Ethics statement}

This study was performed under the regulation of the IRB Committee of The Catholic University of Korea (No. MIRB20200609-004). This research adhered to the tenets of the Declaration of Helsinki. 


\section{Rapid diagnostic test (RDT)}

RDT data with address of subjects from Gyodong-myeon and Samsan-myeon in Ganghwa-gun, Cheorwon-gun, and Goseong-gun was obtained from preceding studies, and the number of patients in each region is summarized in the Table 1 [11-13]. IgG/IgM RDT mounted with recombinant fragment of major surface antigen (SAG1A), GST-linker-SAG1A, were applied to the sera mentioned above [15]. Briefly, $10 \mu \mathrm{l}$ of serum was applied to the RDT sample hole and eluted with RDT buffer, i.e., $0.1 \%$ casein and $1 \%$ Tween 20 in $0.1 \mathrm{M}$ Tris- $\mathrm{HCl}$ buffer ( $\mathrm{pH}$ 8.0) a few seconds later. Reacting bands were read by 3 investigators after $15-20 \mathrm{~min}$, and its density was determined arbitrarily as $0,+,++$, and +++ . Definite reactivity was determined as ++ (mid), while weaker and stronger reactivity compared to it was determined as + (weak) and +++ (strong).

\section{Spatial scan statistic}

Spatial scan statistics continually change the size and location of predefined shaped windows and calculate the relative risk of within and outside the window for the region of interest, identifying clusters with relatively high risk compared to other regions [16]. SaTScan, proposed by Kulldorff (citation) based on the scan statistic, consider windows of each region by gradually increasing its size and allowing the overlap between them. For every window considered, it calculates the likelihood ratio and obtains the maximum likelihood ratio to test the hypothesis that risk of current region of interest is not different from that of outside region. Since the closed form of the distribution of the statistic is not available, SaTScan employs the Monte Carlo (MC) method to conduct the test. It consists of 3 steps as follows. First, generate multiple datasets under the null hypothesis that the cluster does not exist (relative risk $(\mathrm{RR})=1$, the risk is equal in all areas). Second, calculate the test statistic from each of these random repetitive datasets and ranks those in order. Last, set the test statistic as the upper alpha \% of sequence of ordered test statistics in the previous step, and use the value to conduct the test. Null hypothesis is rejected under a significant level of alpha \% if the test statistic calculated from the real data is bigger than the value set by MC simulation. It is also possible to calculate the $P$-value by comparing the test statistic from the real data and the sequence of test statistics from MC simulation [17-20].

Shown in Table 1, a valuable cluster analysis of IgM-RDT cannot be obtained as the incidence of positive patients in IgM-RDT is significantly low, thus the analysis was only per-
Table 1. Prevalence of Toxoplasma gondii-specific antibodies in the residents' sera detected using IgM-RDT and IgG-RDT in Ganghwa-gun, Cheorwon-gun, and Goseong-gun in 2019

\begin{tabular}{lccc}
\hline \multirow{2}{*}{ Location } & \multicolumn{3}{c}{ No. of sera } \\
\cline { 2 - 4 } & Eaxamined & lgM-positive (\%) & lgG-positive (\%) \\
\hline Ganghwa-gun & & & \\
Gyodong-myeon & 255 & $3(1.2)$ & $58(22.7)$ \\
Samsan-myeon & 299 & $4(1.3)$ & $98(32.8)$ \\
Cheorwon-gun & 683 & $8(1.2)$ & $130(19.0)$ \\
Goseong-gun & 306 & $5(1.6)$ & $109(35.6)$ \\
\hline
\end{tabular}

formed on IgG-RDT. IgG-RDT measurement results were recorded in 4 discrete levels (at $0,+,++$, and +++ ) and the proportion of positive patients is above $19.0 \%$. Based on the observations, we choose to employ Bernoulli SaTScan model by dividing IgG-RDT results into positive and negative. For the all tests throughout the paper, we used $5 \%$ of significance level. The base point of each Ri shall be the latitude and longitude coordinates of the village hall (or community center) if there is no Ri office. If there are several halls, the coordinates of the midpoint were used. The window size is set to $25 \%, 15 \%$ per annum for the data in Gyodong-myeon and Samsan-myeon in Ganghwa-gun, Cheorwon-gun, and Goseong-gun. In most cases, the upper cluster region and the relative risk were the same when the maximum allowed window was $15 \%$ and $25 \%$, while the $P$-value was lower when it was $15 \%$. In Table 2 to Table 4, relative risk indicates how much the risk of the cluster and surrounding areas varies. Relative risk is an indicator of how many times positive patients will occur in the cluster than a non-cluster. Log likelihood indicates how many times the number of expected patients than observed. The greater the $P$ value, the less significant the cluster is. Conversely, the smaller the $P$-value, the more certain the area is a cluster. The R 3.6.3 program was used to visualize clusters on the map.

\section{RESULTS}

Using SaTScan, the average annual clusters of each region of Gyodong-myeon and Samsan-myeon in Ganghwa-gun, Cheorwon-gun, and Goseong-gun were analyzed and marked on the map according to the relative risk of each cluster. A cluster with a $P$-value less than 0.05 is significant, but all clusters are plotted for visual comparison. If the cluster contains multiple regions, all are marked. 
Table 2. Clusters detected from Gyodong-myeon and Samsan-myeon in Ganghwa-gun by maximum allowed window in 2019

\begin{tabular}{|c|c|c|c|c|c|c|}
\hline Max window & Cluster & Location & Case & LLR & $\mathrm{RR}$ & $P$-value \\
\hline \multicolumn{7}{|c|}{ Gyodong-myeon } \\
\hline \multirow[t]{2}{*}{$25 \%$} & 1 & Jiseok-ri, Insa-ri & 19 & 4.47 & 2.07 & 0.024 \\
\hline & 2 & Dongsan-ri & 6 & 0.22 & 1.29 & 0.996 \\
\hline \multirow[t]{2}{*}{$15 \%$} & 1 & Insa-ri, Jiseok-ri & 19 & 4.56 & 2.07 & 0.016 \\
\hline & 2 & Dongsan-ri & 6 & 0.22 & 1.29 & 0.980 \\
\hline \multicolumn{7}{|c|}{ Samsan-myeon } \\
\hline \multirow[t]{2}{*}{$25 \%$} & 1 & Seokmo-ri & 27 & 9.56 & 2.21 & $<0.001$ \\
\hline & 2 & Seokpo-ri & 14 & 0.54 & 1.27 & 0.761 \\
\hline $15 \%$ & 1 & Seokpo-ri & 14 & 0.54 & 1.27 & 0.379 \\
\hline
\end{tabular}

Table 3. Clusters detected from Cheorwon-gun by maximum allowed window in 2019

\begin{tabular}{lclcccc}
\hline Max window & Cluster No. & \multicolumn{1}{c}{ Location } & Case & LLR & RR & $P$-value \\
\hline $25 \%$ & 1 & Yangji-ri, lgil-ri & 27 & 4.91 & 1.89 & 0.013 \\
& 2 & Saengchang-ri, Dochang-ri & 18 & 0.78 & 1.34 & 0.745 \\
& 3 & Daema-ri & 20 & 0.31 & 1.19 & 0.948 \\
$15 \%$ & 1 & Yangji-ri, lgil-ri & 27 & 4.91 & 1.89 & 0.010 \\
& 2 & Saengchang-ri, Dochang-ri & 18 & 0.78 & 1.34 & 0.697 \\
& 3 & Daema-ri & 20 & 0.31 & 1.19 & 0.928 \\
\hline
\end{tabular}

Table 4. Clusters detected from Goseong-gun by maximum allowed window in 2019

\begin{tabular}{lclcccc}
\hline Max window & Cluster No. & \multicolumn{1}{c}{ Location } & Case & LLR & RR & $P$-value \\
\hline $25 \%$ & 1 & Madal-ri, Baebong-ri & 16 & 3.62 & 1.79 & 0.050 \\
& 2 & Jukjeong-ri & 12 & 0.53 & 1.29 & 0.847 \\
& 3 & Chodo-ri & 17 & 0.01 & 1.03 & 0.998 \\
$15 \%$ & 1 & Madal-ri, Baebong-ri & 16 & 3.62 & 1.79 & 0.042 \\
& 2 & Jukjeong-ri & 12 & 0.53 & 1.29 & 0.785 \\
\hline
\end{tabular}

\section{Ganghwa-gun}

In Gyodong-myeon with the 25\% maximum allowed window, Jiseok-ri and Insa-ri were the most likely cluster and at a significant level of 5\%, with loglikelihood ratio $=4.47094$, relative risk $=2.066$, and $P<0.05$ ), indicating the risk in Jiseok-ri and Insa-ri was 2.066 times higher than other area (Table 2). Dongsan-ri was the secondary cluster $(P>0.05)$. With the $15 \%$ maximum allowed window, Insa-ri and Jiseok-ri were the most likely-cluster and at a significant level of $5 \%$, with loglikelihood ratio $=4.556293$, relative risk $=2.066$, and $P<0.05$ ), indicating the risk in Insa-ri and Jiseok-ri was 2.066 times higher than other area. Dongsan-ri was the secondary cluster $(P>0.05)$. Jiseok-ri and Insa-ri were the cluster in Gyodongmyeon in 2019 (Fig. 1A).

In Samsan-myeun with the 25\% maximum allowed window, Seokmo-ri was significant as the most likely cluster with loglikelihood ratio $=9.559452, \mathrm{RR}=2.211$ and $P$-value was lower than 0.001, indicating the risk in Seokmo-ri was 2.21 times higher than other area (Table 2). The second cluster was
Seokpo-ri $(P>0.05)$. When the window was $15 \%$, Seokpo-ri was the first cluster with $P$-value $>0.05$, not significant cluster. In the results of the SaTScan analysis in Samsan-myeon, Seokmo-ri was the most likely cluster at the maximum window 25\% (Fig. 1B). We noticed that the results of detected cluster could be changed by a size of window used in analysis. In this case, Seokmo-ri was bigger than $15 \%$ of whole Samsan-myeon area, which causes a huge difference between 2 analyses using different window size. It was worth to mention that we suggest to make a decision on a window size based on the expert's perspective and the scientific question that we want to know the answer, not only based on the numbers from statistical analyses.

\section{Cheorwon-gun}

With the 25\% maximum allowed window, Yangij-ri and Igil-ri were the most likely cluster and at a significant level of 5\%, with loglikelihood ratio $=4.909$, relative risk $=1.886$, and $P<0.05$, indicating the risk in Yangji-ri and Igil-ri was 1.886 
A

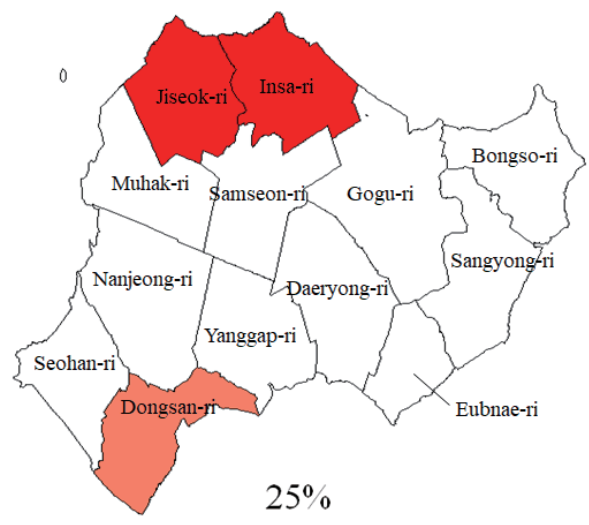

B

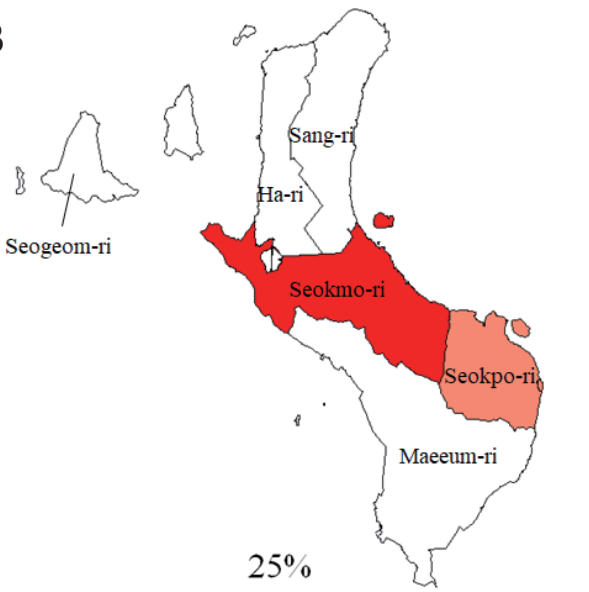

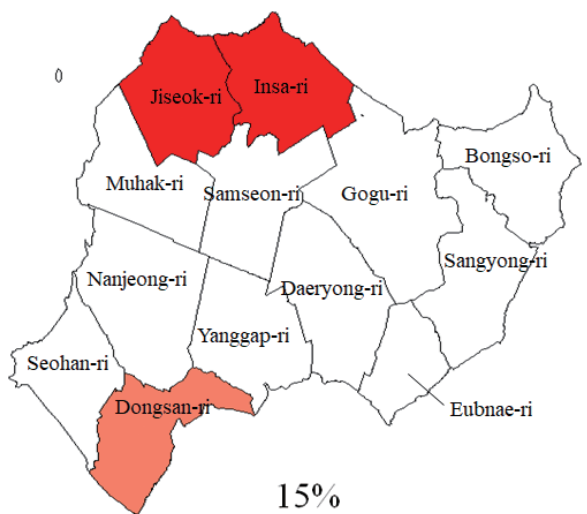

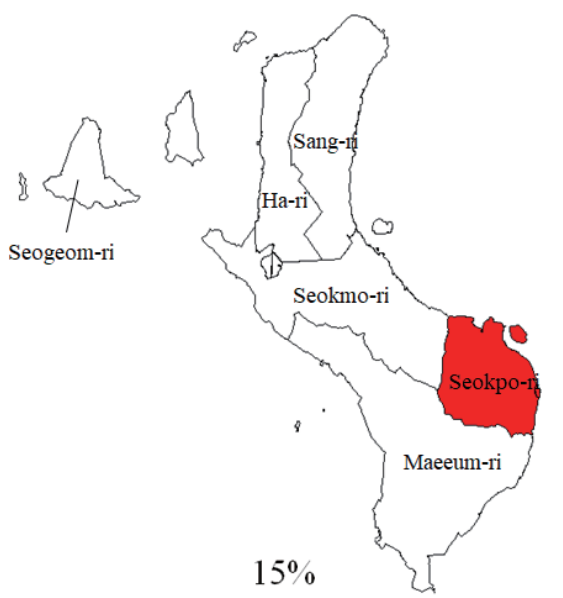

Fig. 1. Toxoplasmosis clusters in Ganghwa-gun by maximum window size in 2019. (A) showed the cluster of Gyodong-myeon and (B), that of Samsan-myeon. The higher the relative risk, the red the darker.

times higher than other area (Table 3). Saengchang-ri and Dochang-ri were the secondary cluster, Daema-ri was the 3rd $(P>0.05)$. When the window was $15 \%$, Yangii-ri and Igil-ri were a most likely cluster with loglikelihood ratio $=4.909$, relative risk $=1.886$, and $P<0.05$. Saengchang-ri and Dochang-ri were the secondary cluster, Daema-ri was the $3 \mathrm{rd}(P>0.05)$. Yangji-ri and Igil-ri were the most likely cluster in Cheorwongun at both window sizes (Fig. 2).

\section{Goseong-gun}

According to analysis on the Goseong-gun cluster focused on Hyeonnae-myeon, Madal-ri and Baebong-ri were the most likely cluster at a significant level of $5 \%$, with loglikelihood ratio $=3.622$, relative risk $=1.793$, and $P=0.05$, indicating the risk in Madal-ri and Baebong-ri was 1.793 times higher than other area (Table 4), at 25\% window size. Jukjeong-ri was the secondary cluster, Chodo-ri was the 3rd $(P>0.05)$. When the window was $15 \%$, Madal-ri and Baebong-ri were the most likely cluster with loglikelihood ratio $=3.622$, relative risk= 1.793 , and $P$-value $=0.042(<0.05)$, indicating the risk in Madal-ri and Baebong-ri was 1.793 times higher than other area. Jukjeong-ri was the secondary cluster $(P>0.05)$. Madal-ri and Baebong-ri were the most likely cluster in Goseong-gun at both window size (Fig. 3).

\section{DISCUSSION}

This study provided clusters with a high risk of toxoplasmosis in Gyodong-myeon and Samsan-myeon, Ganghwa-gun, Cheorwon-gun, Goseong-gun, Korea through spatial scan analysis in 2019. At the maximum allowed window size of $25 \%$ and $15 \%$, it was shown as Jiseok-ri and Insa-ri are the most likely cluster in Gyodong-myeon. In Samsan-myeon, the most likely cluster is Seokmo-ri. Yangii-ri and Igil-ri are the most likely cluster in Cheorwon-gun. Madal-ri and Baebong-ri are the most likely cluster in Goseong-gun. SaTScan recom- 


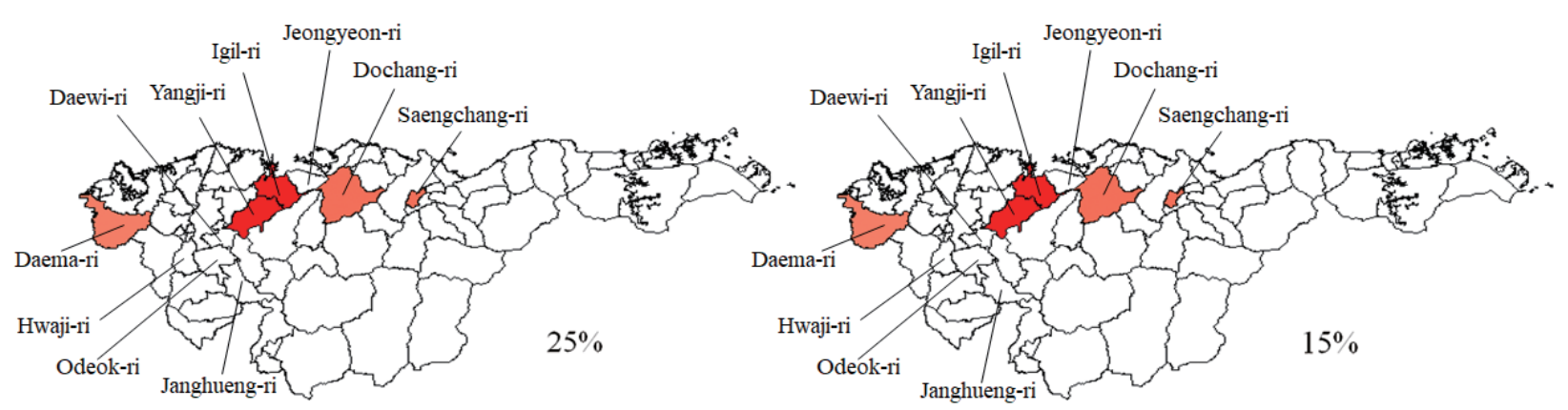

Fig. 2. Toxoplasmosis clusters in Cheorwon-gun by maximum window size in 2019. The higher the relative risk, the red the darker.
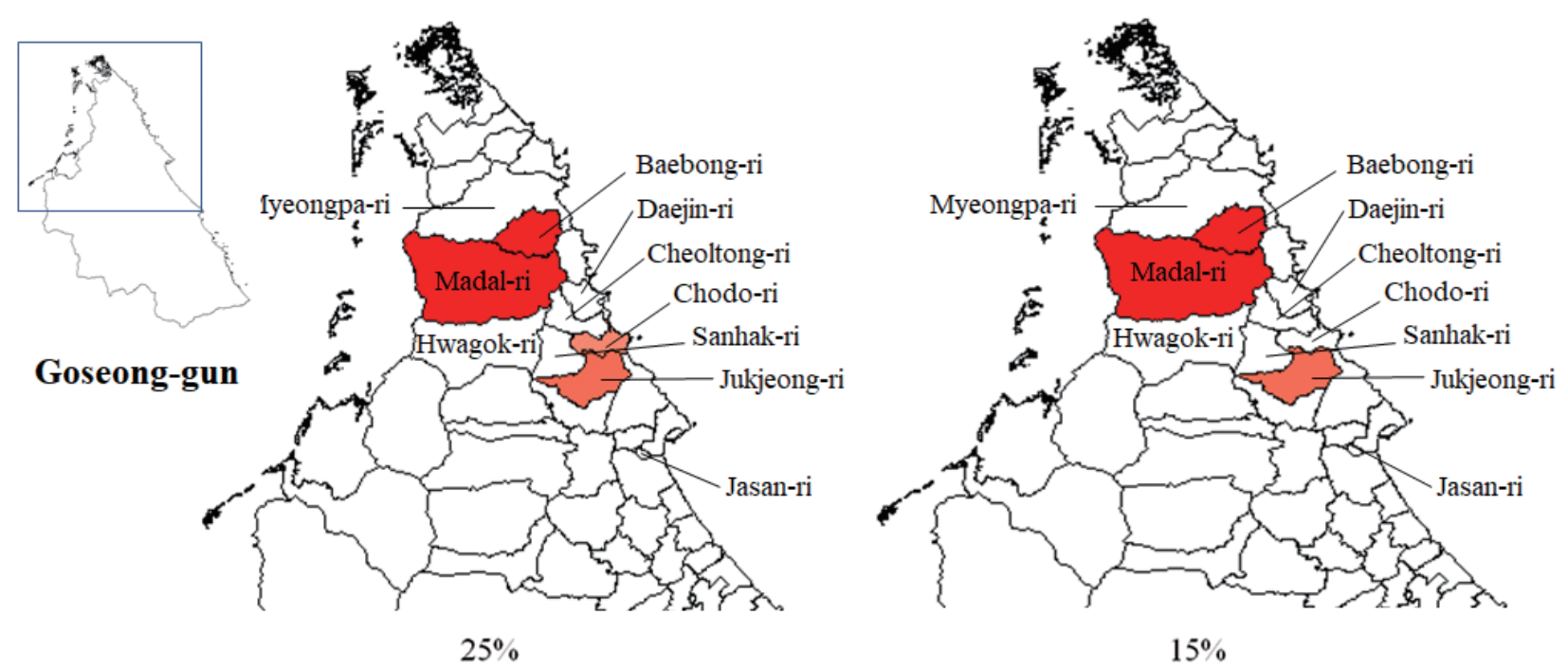

Fig. 3. Toxoplasmosis clusters in Goseong-gun by maximum window size in 2019. The higher the relative risk, the red the darker.

mends a maximum window size less than or equal to $50 \%$ of total population. Due to the small population of the study area, maximum windows sizes were set at $25 \%$ and $15 \%$. In Gyodong, Cheorwon, and Goseong, there is no difference between clusters when the window size is $25 \%$ and $15 \%$. This indicates that clusters in the 3 regions are constant regardless of window size changes. On the other hand, in Samsan, the cluster is different when the window size is $25 \%$ and $15 \%$. Considering $25 \%$ of the total population, Seokmo-ri becomes a cluster when Seokpo-ri is a cluster of $15 \%$ window size.

Gyodong-myeon is the northernmost island of the Military Demarcation Line. Most of the islands are surrounded by iron fences, most residents are engaged in agriculture rather than fishing. There is a wharf on the east side of the island, and recently it is connected to Ganghwa-do island by yeonpyeong bridge. Gyodong-island is a region where toxoplastic lymphadenitis had a group outbreak in residents who ingested pork raised at home in 1997 [7]. Micro cluster in the island is located in the village in the northwest, and toxoplasmic enironment by cats is maintained until now. It is estimated that the better conditions are presented for the spread of infectious sources due to the recent introduction of organic farming methods in agricultural areas. Samsan-myeon is the administrative district of Seokmodo-do. Micro-clusters are formed in the central area of the island near the wharf in the southwest and recently connected to the main island of Gangwha-do by the bridge.

Cheorwon-gun is located near border and has well preserved nature. Goseomg-gun is also located near border with demilitized zone but different from those regions with big mountains of Taeback, which showed different isolated region geographically. Even though the geographical characteristics the prevalence of toxoplasmosis revealed to be almost same.

Therefore, cluster analysis in relatively narrow islands in this 
study can estimate whether the source of $T$. gondii is oocyst mediated by cats, or tissue-cyst mediated by food and mainly pork, and can exclude T. gondii infection environments from farming structures. Also, we could observe that perspective of experts and practitioners on the study is very important. The size of window affect the analysis result and it seems to be reasonable to select the appropriate window size based on the purpose of the study, different perspectives of researchers, etc. The findings can be used to monitor and prevent toxoplasmosis infections occurring in vulnerable areas.

\section{CONFLICT OF INTEREST}

The authors declare that they have no competing interests.

\section{REFERENCES}

1. Black MW, Boothroyd JC. Lytic cycle of Toxoplasma gondii. Microbiol Mol Biol Rev 2000; 64: 607-623. https://doi.org/10.1128/ mmbr.64.3.607-623.2000

2. Furtado JM, Smith JR, Belfort R Jr, Gattey D, Winthrop KL. Toxoplasmosis: a global threat. J Glob Infect Dis 2011; 3: 281-284. https://doi.org/10.4103/0974-777X.83536

3. Tenter AM, Heckeroth AR, Weiss LM. Toxoplasma gondii: from animals to humans. Int J Parasitol 2000; 30: 1217-1258. https:// doi.org/10.1016/s0020-7519(00)00124-7

4. Montoya JG, Remington JS. Management of Toxoplasma gondii infection during pregnancy. Clin Infect Dis 2008; 47: 554-566. https://doi.org/10.1086/590149

5. Song KJ, Shin JC, Shin HJ, Nam HW. Seroprevalence of toxoplasmosis in Korean pregnant women. Korean J Parasitol 2005; 43: 69-71. https://doi.org/10.3347/kjp.2005.43.2.69

6. Weiss LM, Dubey JP. Toxoplasmosis: a history of clinical observations. Int J Parasitol 2009; 39: 895-901. https://doi.org/10.1016/ j.ijpara.2009.02.004

7. Choi WY, Nam HW, Kwak NH, Huh W, Kim YR, Kang MW, Cho SY, Dubey JP. Foodborne outbreaks of human toxoplasmosis. J Infect Dis 1997; 175: 1280-1282. https://doi.org/10.1086/593702

8. Kim MH, Choi YK, Park YK, Nam HW. A toxoplasmic uveitis case of a 60-year-old male in Korea. Korean J Parasitol 2000; 38 : 29-31. https://doi.org/10.3347/kjp.2000.38.1.29

9. Park YH, Han JH, Nam HW. Clinical features of ocular toxoplas- mosis in Korean patients. Korean J Parasitol 2011; 49: 167-171. https://doi.org/10.3347/kjp.2011.49.2.167

10. Ahn HJ, Cho PY, Ahn SK, Kim TS, Chong CK, Hong SJ, Cha SH, Nam HW. Seroprevalence of toxoplasmosis in the residents of Cheorwon-gun, Gangwon-do, Korea. Korean J Parasitol 2012; 50: 225-227. https://doi.org/10.3347/kjp.2012.50.3.225

11. Kim YH, Lee JH, Ahn SK, Kim TS, Hong SJ, Chong CK, Ahn HJ, Nam HW. High seroprevalence of toxoplasmosis detected by RDT among the residents of Seokmo-do (Island) in GanghwaGun, Incheon City, Korea. Korean J Parasitol. 2017; 55: 9-13. https://doi.org/10.3347/kjp.2017.55.1.9

12. Kim YH, Lee JH, Ahn SK, Kim TS, Hong SJ, Chong CK, Ahn HJ, Nam HW. Seroprevalence of toxoplasmosis with ELISA and rapid diagnostic test among residents in Gyodong-do, Inchon city, Korea: a four-year follow-up. Korean J Parasitol. 2017; 55: 247254. https://doi.org/10.3347/kjp.2017.55.3.247

13. Kim YH, Lee JH, Kim YE, Ahn SK, Kim TS, Hong SJ, Chong CK, Ahn HJ, Nam HW. Seroprevalence of toxoplasmosis detected by RDT in residents near the DMZ (demilitarized zone) of Cheorwon-gun, Gangwon-do, Korea. Korean J Parasitol 2017; 55.4: 385-389. https://doi.org/10.3347/kjp.2017.55.4.385

14. Hong SJ, Chong CK, Lee K, Kim TS, Hong YP, Ahn HJ, Kim HY, Ko AR, Kim YJ, Nam HW. Maintained seroprevalence of toxoplasmosis among the residents of Jeju island, Korea. Korean J Parasitol 2011; 49: 309-311. https://doi.org/10.3347/kjp.2011.49.3.309

15. Song KJ, Yang Z, Chong CK, Kim JS, Lee KC, Kim TS, Nam HW. A rapid diagnostic test for toxoplasmosis using recombinant antigenic N-terminal half of SAG1 linked with intrinsically unstructured domain of gra2 protein. Korean J Parasitol 2013; 51: 503-510. https://doi.org/10.3347/kjp.2013.51.5.503

16. Kulldorff M. A spatial scan statistic. Commun Stat Theory Methods 1997; 26:1481-1496. https://doi.org/10.1080/03610929708831995

17. Kulldorff M. SaTScan TM: User Guide for version 9.6. 2018. [Internet]. Available from: https://www.satscan.org/techdoc.html.

18. Han JH, Lee MJ. Cancer cluster detection using scan statistic. J Korean Data Inf Sci Soc 2016; 27:1193-1201 (in Korean). https:// doi.org/10.7465/jkdi.2016.27.5.1193

19. Kulldorff M. An isotonic spatial scan statistic for geographical disease surveillance. J Nat Inst of Public Health 1999; 48: 94101. https://www.satscan.org/papers/ku-JNIPH1999.pdf

20. Azage M, Kumie A, Worku A, Bagtzoglou AC. Childhood diarrhea exhibits spatiotemporal variation in northwest Ethiopia: a SaTScan spatial statistical analysis. PLoS One 2015;10: e0144690. https://doi.org/10.1371/journal.pone.0144690 